\title{
Serum cytokine concentrations and acute graft-versus-host disease after allogeneic peripheral blood stem cell transplantation: Concurrent measurement of ten cytokines and their respective ratios using cytometric bead array
}

\author{
NOBUHARU FUJII ${ }^{1}$, AKIO HIRAKI ${ }^{2}$, KEISUKE AOE $^{2,3}$, TOMOYUKI MURAKAMI ${ }^{3}$, KAZUMA IKEDA ${ }^{1}$, \\ KOZO MASUDA $^{1}$, KEITARO MATSUO ${ }^{4}$, KATSUJI SHINAGAWA ${ }^{1}$, FUMIHIKO ISHIMARU ${ }^{1}$, \\ KAZURO SUGI ${ }^{3}$, ZBIGNIEW DARZYNKIEWICZ ${ }^{5}$ and MITSUNE TANIMOTO ${ }^{1}$ \\ ${ }^{1}$ Department of Hematology, Oncology and Respiratory Medicine, Okayama University Medical School, \\ Okayama; Departments of ${ }^{2}$ Respiratory Medicine and ${ }^{3}$ Clinical Research, NHO Sanyo National Hospital, \\ Respiratory Disease Center, Ube, Yamaguchi; ${ }^{4}$ Division of Epidemiology and Prevention, Aichi Cancer Center \\ Research Institute, Nagoya, Aichi, Japan; ${ }^{5}$ Brander Cancer Research Institute, New York Medical College, NY 10532, USA
}

Received November 28, 2005; Accepted January 16, 2006

\begin{abstract}
Both inflammatory and anti-inflammatory cytokines have been reported to be associated with acute graft-versushost disease (aGVHD). However, their role and possible mutual interactions during aGVHD are not well understood. Eight patients with aGVHD and eight without who had undergone allogeneic HLA-identical peripheral blood stem cell transplantation were studied. The patients had no other complications known to affect serum concentration of cytokines, including infection. Serum concentrations of IL-1ß, IL-2, IL-4, IL-5, IL-6, IL-8, IL-10, IL-12p70, TNF- $\alpha$ and IFN- $\gamma$ were concurrently measured by a new technique, the cytometric bead array (CBA). We found that serum concentrations of IL-5, IL-6 and IL-10 were significantly higher in patients with aGVHD than in patients without it. By ratiometric analysis, the ratios of IL-5/IL-2, IL-5/IL-4, IL-6/ IL-4 in patients with aGVHD were increased compared to the patients with no evidence of aGVHD. ROC analysis demonstrated that the ratio of IL-5/IL-4 was the most sensitive parameter associated with aGVHD. The second best marker of aGVHD was increased IL-5 concentration. Thus, our results indicate that the ratio of a particular cytokine/ cytokine could be a potential diagnostic marker for aGVHD, more sensitive that the serum level of a given cytokine. This
\end{abstract}

Correspondence to: Dr Keisuke Aoe, Department of Respiratory Medicine and Clinical Research, NHO Sanyo National Hospital Respiratory Disease Center, 685 Higashi-kiwa, Ube, Yamaguchi 755-0241, Japan

E-mail: aoe@sanyou-dr.jp

Key words: diagnostic marker, flow cytometry observation is consistent with a cross-talk among some cytokines and their possible interactions via respective receptors on cytokine-producing cells; these interactions may play an important role in pathogenesis of aGVHD. Further studies including a large number of patients and concurrent measurement of a variety of cytokines are needed to fully assess the diagnostic value of the cytokine ratiometric analysis. The CBA methodology provides a convenient and useful tool in such studies.

\section{Introduction}

Acute graft-versus-host disease (aGVHD), which occurs in $>30 \%$ of patients who undergo allogeneic stem cell transplantation is still one of the major contributors to transplantrelated mortality (1). Thus, to reduce the rate of transplantrelated mortality, management of aGVHD is a critical clinical problem.

Cytokines are proteins with relatively low molecular weight that are secreted by cells in reaction to a variety of different stimuli. Cytokines are important immunoregulators and act as key mediators of the host response to various infectious, inflammatory, and immunologic challenges (2-4). A variety of cytokines have been demonstrated to be associated with aGVHD. Accumulating evidence has shown that inflammatory cytokines including tumor necrosis factor (TNF)- $\alpha$, interleukin (IL)-1 or IL-6 play primary roles in activating T cells leading to aGVHD (5). Th1 cytokines including IL-2, interferon (IFN) $-\gamma$ induced by inflammatory cytokines also cause tissue destruction in aGVHD (6). On the other hand, the elevation of serum anti-inflammatory cytokines such as IL-10 or transforming growth factor-ß1 (TGF-ß1) has also been reported (7). It is likely that within the complex mechanism of the cytokine network during aGVHD that altered expression of a particular cytokine (e.g. cytokine a) may modulate the expression of another cytokine (e.g. cytokine b) and thus 
Table I. Patient charactereristics.

\begin{tabular}{|c|c|c|c|c|c|c|c|c|}
\hline & Disease & Age & Sex & Disease status & $\begin{array}{c}\text { No. of CD34+ } \\
\quad \times 10^{6} / \mathrm{kg}\end{array}$ & Conditioning & $\begin{array}{c}\text { aGVHD } \\
\text { prophylaxis }\end{array}$ & $\begin{array}{c}\text { aGVHD } \\
\text { (Grade; organ) }\end{array}$ \\
\hline 1 & AML(M2) & 18 & $\mathrm{~F}$ & Primary Ref & 4 & BU/CY/Flu & CsA/sMTX & III; Gl \\
\hline 2 & NHL(MCL) & 67 & $\mathrm{M}$ & Primary Ref & 1.8 & $\mathrm{CY} / \mathrm{Flu}$ & CsA/sMTX & I; Skin \\
\hline 3 & NHL(FL) & 58 & $\mathrm{M}$ & Ref/2nd T & 4.52 & $\mathrm{CY} / \mathrm{Flu}$ & CsA/sMTX & III; Skin, GI \\
\hline 4 & NHL(DL) & 28 & M & Primary Ref & 4.35 & $\mathrm{CY} / \mathrm{TBI}$ & CsA/sMTX & III; Skin, GI, Liver \\
\hline 5 & AML(M2) & 30 & $\mathrm{~F}$ & $2 \mathrm{CR}$ & 5.53 & $\mathrm{CY} / \mathrm{TB} 1$ & CsA/sMTX & II; Skin, GI \\
\hline 6 & AML(M2) & 58 & M & $1 \mathrm{CR}$ & 2.9 & $\mathrm{BU} / \mathrm{CY}$ & CsA/sMTX & II; Skin, GI \\
\hline 7 & MDS/AML & 55 & $\mathrm{~F}$ & Relapse/Ref & 2.74 & $\mathrm{BU} / \mathrm{CY}$ & FK/sMTX & III; Skin, Gl, Liver \\
\hline 8 & ALL(L2) & 40 & $\mathrm{~F}$ & $1 \mathrm{CR}$ & 3.83 & L-PAM/TBI & CsA/sMTX & I; Skin \\
\hline 9 & CML(CP1) & 35 & $\mathrm{~F}$ & $1 \mathrm{CP}$ & 10 & $\mathrm{BU} / \mathrm{CY}$ & CsA/sMTX & - \\
\hline 10 & CML(CP1) & 18 & $\mathrm{M}$ & $1 \mathrm{CP}$ & 7.97 & $\mathrm{BU} / \mathrm{CY}$ & CsA/sMTX & - \\
\hline 11 & AML(M4) & 39 & $\mathrm{~F}$ & Primary Ref & 4 & $\mathrm{CY} / \mathrm{Flu}$ & CsA/sMTX & - \\
\hline 12 & CML(CP1) & 35 & $\mathrm{~F}$ & $\mathrm{CP}$ & 6.38 & $\mathrm{BU} / \mathrm{CY}$ & CsA/sMTX & - \\
\hline 13 & $\operatorname{MDS}(\mathrm{RA})$ & 58 & M & No treatment & 1.62 & $\mathrm{CY} / \mathrm{Flu}$ & CsA/sMTX & - \\
\hline 14 & MM & 52 & M & Relapse/Ref & 3.5 & L-PAM/Flu & CsA/sMTX & - \\
\hline 15 & AML(M2) & 62 & $\mathrm{~F}$ & $1 \mathrm{CR}$ & 2.19 & $\mathrm{CY} / \mathrm{Flu}$ & CsA/sMTX & - \\
\hline 16 & AML(M6a) & 18 & $\mathrm{M}$ & $1 \mathrm{CR}$ & 4.67 & $\mathrm{CY} / \mathrm{TBI}$ & CsA/sMTX & - \\
\hline
\end{tabular}

AML, acute myelogenous leukemia; NHL, non-Hodgkin lymphoma; MCL, mantle cell lymphoma; FL, follicular lymphoma; DL, diffuse large B cell lymphoma; MDS/AML, myelodysplastic syndrome/overt leukemia; ALL, acute lymphoblastic leukemia; CML, chronic myeloid leukemia; CP1, first chronic phase; RA, refractory anemia; MM, multipte myeloma; F, female; M, male; Ref, refractory; 2nd T, second transplantation; CR, complete remission; BU, busulfan; CY, cyclophoshamide; Flu, fludarabine; TBI, total body irradiation; L-PAM, melphalan; CsA, cyclosporine A; MTX, methotrexete; FK, FK506(tacrolimus); GI, gastrointestine.

there is a mutual cause-effect interaction between them. Such interaction is expected to be reflected by the consistently altered (cytokine a)/(cytokine b) ratio, the alteration more pronounced than a change in expression of either of these cytokines alone. This kind of cytokine-to-cytokine interaction may play important roles in both development and regulation of aGVHD. However, no report on concurrent measurement of the concentration of multiple cytokines and the examination of their relationship with the occurrence of aGVHD exist.

We studied 16 patients to measure serum concentrations of IL-1ß, IL-2, IL-4, IL-5, IL-6, IL-8, IL-10, IL-12, TNF- $\alpha$ and IFN- $\gamma$ with a newly developed technique, cytometric bead array (CBA). The CBA assay uses uniform-size microparticle-based flow cytometry to measure a panel of human cytokines simultaneously (8). The ability of CBA to measure the concentrations of 10 cytokines from the same serum permits calculation of cytokine ratio and examined the significance for occurrence of aGVHD.

\section{Patients and methods}

Patients. Sixteen patients who had undergone allogeneic HLAidentical peripheral blood stem cell transplantation (alloPBSCT) in Okayama University Hospital from November 1998 to December 2002 were studied. Patient characteristics and transplant procedures are summarized in Table I. Briefly, the median age of the patients was 40 years (range 18-67). The diagnosis included AML (n=7), NHL ( $=3)$, CML $(n=3)$ and others (ALL, MDS, MM). Disease status at transplant consisted of 9 standard-risk [1st complete remission and 1st chronic phase $(\mathrm{CML})]$ patients and 7 high-risk patients. Preparative regimen included 9 conventional [total body irradiation (TBI)/ cyclophosphamide (CY), busulfan/CY, TBI/L-PAM] regimens and 7 fludarabine-based nonmyeloablative regimens. The infused number of PBSC are shown in Table I. For aGVHD prophylaxis, $3 \mathrm{mg} / \mathrm{kg} /$ day cyclosporine A (continuous i.v. from day 1) or tacrolimus $(0.03 \mathrm{mg} / \mathrm{kg} /$ day $)$ with methotrexete [i.v. day $1\left(10 \mathrm{mg} / \mathrm{m}^{2}\right)$, day 3 and day $5\left(7 \mathrm{mg} / \mathrm{m}^{2}\right)$ ] were given. Skin or gut biopsy was performed for histologically confirmation of aGVHD.

Among them, 8 patients did not suffer from acute GVHD. Other 8 patients developed Grade I-III aGVHD (Grade I; 2, Grade II; 2, Grade III; 4). The patients with Grade I aGVHD were not treated against aGVHD. As the treatment of Grade II aGVHD, predonisolone ( $1 \mathrm{mg} / \mathrm{kg} /$ day $)$ was initiated. Patients with any complications except aGVHD including infection were excluded from the present study.

Collection of sera. All blood samples for cytokine determination were collected with a written informed consent. Serum was separated immediately and stored at $-20^{\circ} \mathrm{C}$ until assays were performed.

Serum of the patients with aGVHD was collected prior to the conditioning therapy and just before the initiation of steroid therapy for aGVHD (Grade II-IV). In the patients with 
Table II. Coefficients and p-values in serum concentrations of cytokines.

\begin{tabular}{lcccccccccc}
\hline & IL-1ß & IL-2 & IL-4 & IL-5 & IL-6 & IL-8 & IL-10 & IL-12 & IFN- $\gamma$ & TNF- $\alpha$ \\
\hline IL-1ß & x & NS & NS & NS & NS & NS & NS & NS & NS & NS \\
IL-2 & NS & x & 0.0702 & $\mathbf{0 . 0 0 3 8}$ & 0.0616 & NS & NS & NS & NS & NS \\
IL-4 & NS & NS & x & $\mathbf{0 . 0 0 0 3}$ & $\mathbf{0 . 0 2 0 5}$ & NS & NS & NS & NS & NS \\
IL-5 & 0.0399 & $\mathbf{0 . 0 0 5 3}$ & $\mathbf{0 . 0 5 0 3}$ & x & 0.0313 & 0.0316 & 0.0257 & 0.0829 & 0.0137 & 0.0423 \\
IL-6 & 0.0674 & NS & $\mathbf{0 . 0 3 4 8}$ & NS & x & 0.0373 & NS & 0.0745 & NS & 0.0302 \\
IL-8 & NS & NS & NS & NS & NS & x & NS & NS & NS & 0.0585 \\
IL-10 & 0.0109 & 0.0438 & 0.0143 & NS & NS & 0.0894 & x & 0.0208 & NS & 0.0122 \\
IL-12 & 0.0053 & NS & NS & NS & NS & NS & NS & x & NS & NS \\
IFN- $\gamma$ & 0.0555 & NS & NS & NS & NS & NS & NS & NS & x & NS \\
TNF- $\alpha$ & NS & NS & NS & NS & NS & NS & NS & NS & NS & c \\
\hline
\end{tabular}

Bold type, statistically significant.

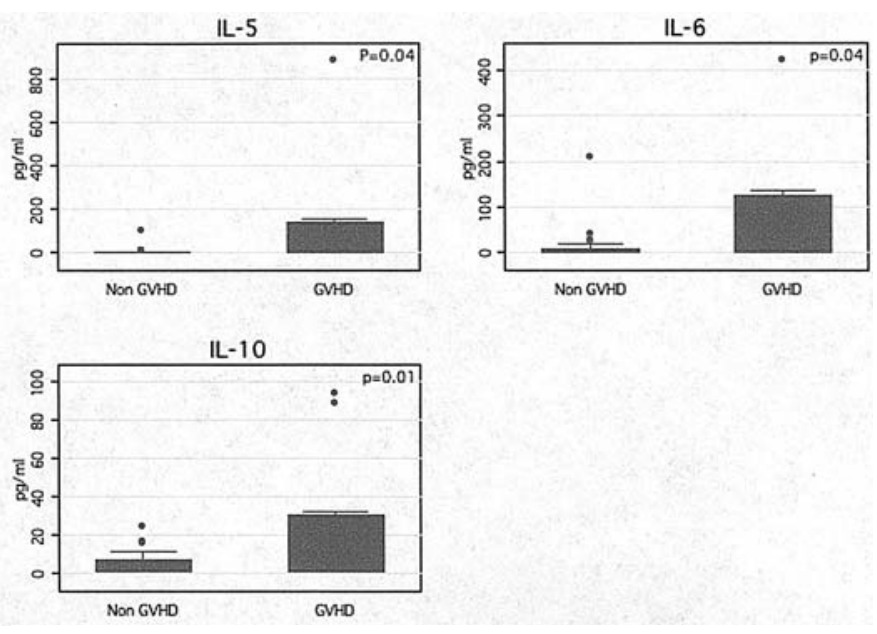

Figure 1. Serum concentrations of L-5, IL-6 and IL-10 in patients with aGVHD and without (A). Patients with aGVHD also exhibited higher serum concentrations of IL-5 (4.63 \pm 18.7 vs. $124.3 \pm 247.4 \mathrm{pg} / \mathrm{ml} ; \mathrm{p}=0.04)$, IL-6 $(12.1 \pm 37.9$ vs. $74.1 \pm 122.3 \mathrm{pg} / \mathrm{ml} ; \mathrm{p}=0.04)$ and IL-10 (4.54 $\pm 6.07 \mathrm{vs}$. $25.4 \pm 33.0 \mathrm{pg} / \mathrm{ml} ; \mathrm{p}=0.01$ ) than patients without.

Grade I aGVHD, serum was collected when aGVHD was histologically confirmed. In the patients without aGVHD, serum was collected between day 30 and 60 , when the patients did not suffer from other transplant-related toxicities including veno-occlusive disease and infections.

Cytometric bead array immunoassay. Serum concentration of IL-1ß, IL-2, IL-4, IL-5, IL-6, IL-8, IL-10, IL-12, TNF- $\alpha$ and IFN- $\gamma$ were simultaneously measured by a CBA kit (Becton Dickinson, San Diego, CA, USA). Briefly, for each serum sample and cytokine standard mixture, $50 \mu 1$ of sample or standard was added to a mixture of $50 \mu 1$ each of capture Abbead reagent and detector Ab-PE reagent. The mixture (150 $\mu \mathrm{l})$ was subsequently incubated for $3 \mathrm{~h}$ at room temperature, and washed to remove unbound detector Ab-PE reagent before data acquisition using flow cytometry.

Flow cytometric analysis. Two-color flow cytometric analysis was performed using FACSan ${ }^{\mathrm{TM}}$ (Becton Dickinson Immunocytometry Systems, San Jose, CA, USA). Data were acquired and analyzed using Becton Dickinson CBA software. Forward vs. side scatter gating was employed to exclude any sample particles other than the $7.5-\mu \mathrm{m}$ polysterene beads. Data were displayed as two-color dot plots (FL-2 vs. FL-3) such that the 6 discrete FL-3 microparticle dye intensities were distributed along the y-axis. Standard curves were plotted (cytokine calibrator concentration vs. FL-2 mean fluorescence intensity) using a four-parameter logistic curvefitting model. Cytokine concentrations were determined from these standard curves. If a sample had a cytokine concentration below the detection limit for the assay, a value of 1 was assigned for that particular cytokine concentration.

Statistical analysis. All analyses were conducted with Stata version 8 (Stata Corp., College Station, TX). Subjects were divided into two groups: those continued without aGVHD throughout transplantation and those that had any grade of aGVHD. Analysis of variance considering repeated measurement within same individual at different time points was applied to examine significance for each cytokine concentration measured by CBA. Area under curve with receiver-operationg-characteristics (ROC) analysis was applied in choosing measures to identify occurrence of aGVHD. $\mathrm{p}<0.05$ was defined as statistically significant.

\section{Results}

Serum cytokine concentrations in patients with and without $a G V H D$. Among the 10 cytokines studied, patients with aGVHD exhibited higher serum concentrations of IL-5 [mean \pm standard deviation (SD); 4.63 \pm 18.7 vs. 124.3 \pm 247.4 $\mathrm{pg} / \mathrm{ml} ; \mathrm{p}=0.04]$ than patients without (Fig. 1A). Patients with aGVHD also exhibited higher serum concentrations of IL-6 $(12.1 \pm 37.9$ vs. $74.1 \pm 122.3 \mathrm{pg} / \mathrm{ml} ; \mathrm{p}=0.04))$ and IL-10 $(4.54 \pm 6.07$ vs. $25.4 \pm 33.0 \mathrm{pg} / \mathrm{ml} ; \mathrm{p}=0.01)$ than patients without (Fig. 1). There was no significant difference between groups with and without aGVHD in the other 7 cytokines (data not shown). There was no significant difference in the tested cytokine levels prior to the conditioning therapy between groups with and without aGVHD (data not shown).

We next examined the relationship ratios of cytokine/ cytokine and occurrence of aGVHD. As summarized in 
A
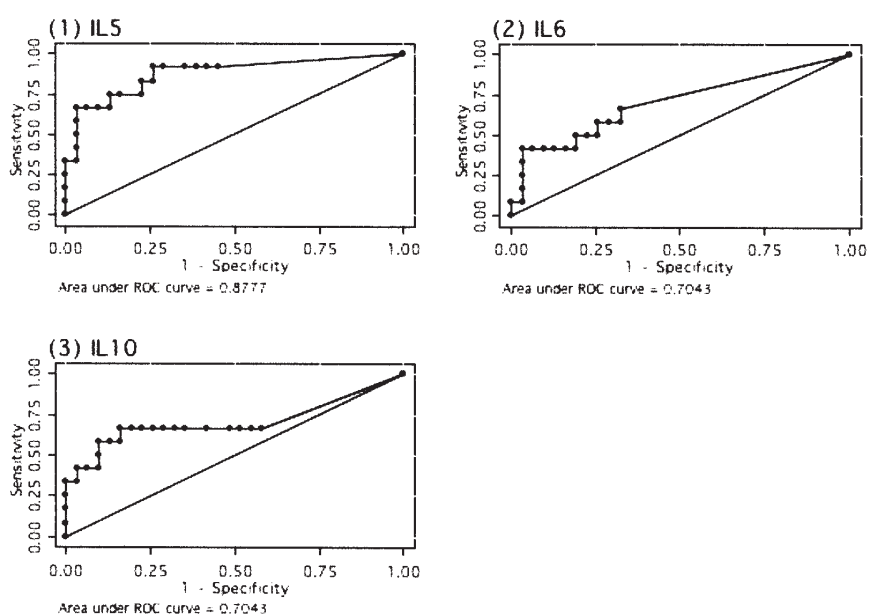

B
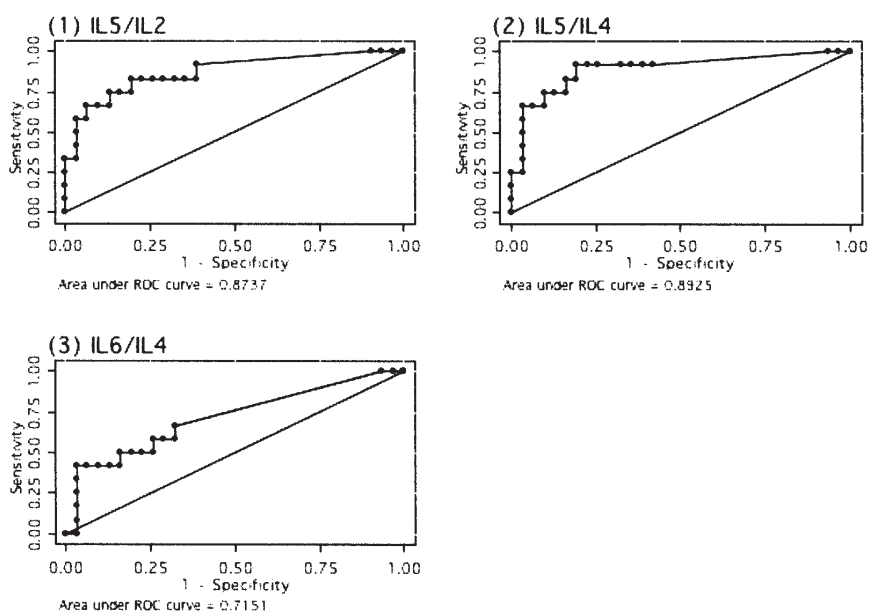

Figure 2. ROC analysis. (A) IL-5 was the most sensitive parameter associated with aGVHD The second best test was IL-6 and IL-10 (AUC, 0.7043). (B) In the ratio of cytokine/cytokine, IL-5/IL-4 ratio has the best ROC curve (AUC, 0.8925). The second best was IL-5/IL-2 ratio (AUC, 0.8737 ) followed by IL-6/IL-4 ratio (AUC, 0.751).

Table II, the ratio of IL-5/IL-2 ( $\mathrm{p}=0.0038)$, IL-5/IL-4 $(\mathrm{p}=0.0003)$, and IL-6/IL-4 $(\mathrm{p}=0.0205)$ were significantly higher in patients with aGVHD than in groups without. No other ratios of cytokine/cytokine were associated with the occurrence of aGVHD.

ROC analysis. To determine the most sensitive and specific indicator for diagnosis of aGVHD, we performed ROC analysis. As shown in Fig. 2A, IL-5 was the most sensitive parameter associated with aGVHD [area under curve (AUC), 0.877]. The second best test was IL-6 and IL-10 (AUC, $0.7043)$. In the ratio of cytokine/cytokine, IL-5/IL-4 ratio has the best ROC curve (AUC, 0.8925). The second best was IL-5/ IL-2 ratio (AUC, 0.8737) followed by IL-6/IL-4 ratio (AUC, 0.751 ; Fig. 2B).

\section{Discussion}

In the present study, we measured 10 cytokines including IL-1ß, IL-2, IL-4, IL-5, IL-6, IL-8, IL-10, IL-12, TNF- $\alpha$ and
IFN- $\gamma$ simultaneously by CBA and examined the relationship between these cytokines and occurrence of aGVHD. Our results showed that serum concentrations of IL-5, IL-6 and IL-10 were elevated in patients with aGVHD compared with patients without it. It is well known that IL-5 is one of the Th2 cytokine essential for eosinophil differentiation and growth. There have been few studies on the relationship between eosinophilia, one of the manifestations of chronic GVHD (9), and aGVHD $(10,11)$, Imoto et al reported that serum IL-5 concentrations were elevated to $>100 \mathrm{pg} / \mathrm{ml}$ in aGVHD patients during manifestation of this complication (10) and we confirmed this in the present study. Taken together, previous studies and our results suggest that IL-5 may play an important role in the development of aGVHD.

Several previous studies have shown that there is a relationship between serum IL-6 $(6,12)$ and IL-10 $(7,13,14)$ and aGVHD. IL-6, one of proinflammatory cytokines, is also known to be elevated during other transplant related complications such as infections and veno-occlusive disease (VOD) $(15,16)$. IL-10 is one of Th2 cytokines to regulate inflammatory response induced by various inflammatory cytokines. Some investigators have also reported that the serum IL-10 level was correlated with the severity of aGVHD $(7,13,14)$. The results obtained in previous studies suggest that IL-6 and IL-10 may also play important roles in the development of aGVHD. The present study confirmed this.

The CBA assay uses uniform-size microparticle-based flow cytometry to measure a panel of human cytokines simultaneously (8). The ability of CBA to measure the concentrations of 10 cytokines from the same serum permits calculation of cytokine/cytokine ratio and to examine the significance for occurrence of aGVHD. We examined the relationship between these cytokine/cytokine ratios and occurrence of aGVHD. Recent studies concerning the diverse cytokine network suggest that the relative balance in abundance between various cytokines may better reflect the net effect of cytokine responses than their absolute concentrations $(17,18)$. Cytokine ratios may be used for the characterization of a variety of disease states such as asthma and atopy (19). In the present study, we found for the first time that the ratio of IL-5/IL-2, IL-5/IL-4, and IL-6/IL-4 were significantly increased in patients with aGVHD. These results suggest that there is a cross-talk among cytokines in which an altered level of one of them provides a feedback for up- or downregulation of another one from the correlated pair, although the role of the observed cytokine ratios in the pathogenesis of aGVHD is unclear. Such feedback interactions are expected in light of the evidence of their pleiotrophic function, their known role in a multitude of different immunologic and metabolic reactions, and that different cell types may synthesize each of them.

The diagnosis of aGVHD is commonly made from observation of skin eruption, diarrhea and elevation of the biliary tract enzyme (20). Management of patients with aGVHD requires an effective treatment plan based on timely and accurate diagnostic information. Thus, a reliable clinical marker providing physicians with rapid and accurate diagnosis of aGVHD is greatly needed. In the present study, ROC analysis demonstrated that the ratio of IL-5/IL-4 has the best ROC curve among 10 cytokines and 45 cytokine/cytokine 
ratios. These results suggest that the ratio of IL-5/IL4 may be a useful indicator for diagnosis of aGVHD.

In summary, we found that serum concentrations of IL-5, IL-6 and IL-10 in patients with acute GVHD were significantly higher than those in patients without. The results suggest that these cytokines may play important roles in pathogenesis of aGVHD. In addition, we also found that the ratios of IL-5/ IL-2, IL-5/IL-4, and IL-6/IL-4 were elevated in patients with acute GVHD compared with those in patients without. The results also suggest that there is cross-talk among cytokines and these interactions may play important roles in pathogenesis and severity of aGVHD. Furthermore, ROC analysis demonstrated that the ratio IL-5/IL-4 was the most sensitive parameter associated with aGVHD among 10 cytokines and the ratios of cytokine/cytokine. Our results suggest that the ratio of cytokine/cytokine could be a potential diagnostic marker for aGVHD. Further studies including a large number of patients and simultaneous measurement of a variety of cytokines using CBA is needed. There were differences in relative abundance of particular cytokines and their respective ratios, among individual patients with aGVHD. It is tempting to speculate that these differences may bear prognostic value and/or be associated with severity of aGVHD. Studies are underway to correlate these variations among patients with the clinical parameters. The CBA methodology provides a convenient tool to investigate these interactions.

\section{References}

1. Ringden O, Horowitz MM, Sondel P, et al: Methotrexate, cyclosporine, or both to prevent graft-versus-host disease after HLA-identical sibling bone marrow transplants for early leukemia? Blood 81: 1094-1101, 1993.

2. Van Deuren M, Dofferhoff AS and van der Meer JW: Cytokines and the response to infection. J Pathol 168: 349-356, 1992.

3. Opal SM and De Palo VA: Anti-inflammatory cytokines. Chest 117: 1162-1172, 2000

4. Nicod LP: Cytokines. 1. Overview. Thorax 48: 660-667, 1993.

5. Ferrara JL, Cooke KR and Teshima T: The pathophysiology of acute graft-versus-host disease. Int J Hematol 78: 181-187, 2003.

6. Imamura M, Hashino S, Kobayashi H, et al: Serum cytokine levels in bone marrow transplantation: synergistic interaction of interleukin-6, interferon-gamma, and tumor necrosis factoralpha in graft-versus-host disease. Bone Marrow Transplant 13: 745-751, 1994.
7. Remberger M, Jaksch M, Uzunel M and Mattsson J: Serum levels of cytokines correlate to donor chimerism and acute graftvs.-host disease after haematopoietic stem cell transplantation. Eur J Haematol 70: 384-391, 2003.

8. Morgan E, Varro R, Sepulveda H, et al: Cytometric bead array: a multiplexed assay platform with applications in various areas of biology. Clin Immunol 110: 252-266, 2004.

9. Kalaycioglu ME and Bolwell BJ: Eosinophilia after allogeneic bone marrow transplantation using the busulfan and cyclophosphamide preparative regimen. Bone Marrow Transplant 14: 113-115, 1994.

10. Imoto S, Oomoto Y, Murata K, et al: Kinetics of serum cytokines after allogeneic bone marrow transplantation: interleukin-5 as a potential marker of acute graft-versus-host disease. Int J Hematol 72: 92-97, 2000.

11. McNeel D, Rubio MT, Damaj G, et al: Hypereosinophilia as a presenting sign of acute graft-versus-host disease after allogeneic bone marrow transplantation. Transplantation 74 : 1797-1800, 2002.

12. Symington FW, Symington BE, Liu PY, Viguet H, Santhanam U and Sehgal PB: The relationship of serum IL-6 levels to acute graft-versus-host disease and hepatorenal disease after human bone marrow transplantation. Transplantation 54: 457-462, 1992.

13. Visentainer JE, Lieber SR, Persoli LB, et al: Serum cytokine levels and acute graft-versus-host disease after HLA-identical hematopoietic stem cell transplantation. Exp Hematol 31: 1044-1050, 2003.

14. Takatsuka H, Takemoto Y, Okamoto T, et al: Predicting the severity of graft-versus-host disease from interleukin-10 levels after bone marrow transplantation. Bone Marrow Transplant 24: 1005-1007, 1999

15. Liem LM, van Houwelingen HC and Goulmy E: Serum cytokine levels after HLA-identical bone marrow transplantation. Transplantation 66: 863-871, 1998.

16. Schwaighofer H, Herold M, Schwarz T, et al: Serum levels of interleukin-6, interleukin-8, and C-reactive protein after human allogeneic bone marrow transplantation. Transplantation 58 : 430-436, 1994.

17. Kenyon NJ, Kelly EA and Jarjour NN: Enhanced cytokine generation by peripheral blood mononuclear cells in allergic and asthma subjects. Ann Allergy Asthma Immunol 85: 115-120, 2000 .

18. Aoe K, Hiraki A, Murakami T, et al: Relative abundance and patterns of correlation among six cytokines in pleural fluid measured by cytometric bead array. Int J Mol Med 12: 193-198, 2003.

19. Maggi E: The TH1/TH2 paradigm in allergy. Immunotechnology 3: 233-244, 1998.

20. Przepiorka D, Weisdorf D, Martin P, et al: 1994 Consensus Conference on Acute GVHD Grading. Bone Marrow Transplant 15: 825-828, 1995. 